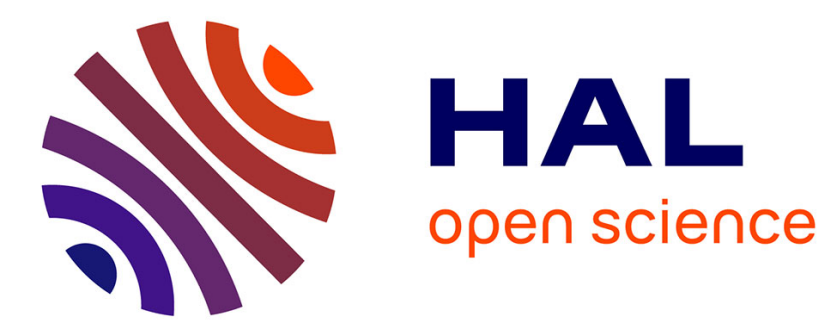

\title{
Benefits of a Brief Physical Activity Programme on Employees' Affective Well-being and Momentary Affective States: A Quasi-Experimental Study
} Anne Courbalay, Quentin Brandet, Delphine Girard, Nicolas Gillet

\section{- To cite this version:}

Anne Courbalay, Quentin Brandet, Delphine Girard, Nicolas Gillet. Benefits of a Brief Physical Activity Programme on Employees' Affective Well-being and Momentary Affective States: A QuasiExperimental Study. Journal of Happiness Studies, In press, 10.1007/s10902-021-00489-9 . hal03548387

\section{HAL Id: hal-03548387 \\ https://hal.science/hal-03548387}

Submitted on 30 Jan 2022

HAL is a multi-disciplinary open access archive for the deposit and dissemination of scientific research documents, whether they are published or not. The documents may come from teaching and research institutions in France or abroad, or from public or private research centers.
L'archive ouverte pluridisciplinaire HAL, est destinée au dépôt et à la diffusion de documents scientifiques de niveau recherche, publiés ou non, émanant des établissements d'enseignement et de recherche français ou étrangers, des laboratoires publics ou privés. 
Physical activity and employees' affective well-being

Running head: Physical activity and employees' affective well-being

\section{Benefits of a Brief Physical Activity Programme on Employees' Affective Well-being and}

\section{Momentary Affective States: A quasi-experimental study}

\section{Anne Courbalay}

APCoSS, Institut de Formation en Education Physique et en Sport d'Angers (IFEPSA), UCO, Les Ponts de Cé, France anne.courbalay@uco.fr

Quentin Brandet

APCoSS, Institut de Formation en Education Physique et en Sport d'Angers (IFEPSA), UCO, Les Ponts de Cé, France

\section{Delphine Girard}

APCoSS, Institut de Formation en Education Physique et en Sport d'Angers (IFEPSA), UCO, Les Ponts de Cé, France

\section{Nicolas Gillet}

EE 1901 Qualité de Vie et Santé Psychologique (QUALIPSY), Université de Tours, UFR Arts et Sciences Humaines, Département de psychologie, Tours, France

This is the prepublication version of the following manuscript:

Courbalay, A., Brandet, Q., Girard, D., \& Gillet, N. (in press). Benefits of a brief physical activity programme on employees' affective well-being and momentary affective states: A quasi-experimental study. Journal of Happiness Studies. Early view. https://doi.org/10.1007/s10902-021-00489-9

(C) 2022. This paper is not the copy of record and may not exactly replicate the authoritative document published in Journal of Happiness Studies. 
Physical activity and employees' affective well-being

\begin{abstract}
The present study examined the effects of a four-week physical activity (PA) programme on employees' general affective well-being the acute effects of fitness and stretching sessions on momentary affective states. Participants were divided between an experimental $(n=54)$ and a comparison group $(n=57)$. After one month, only employees in the experimental group increased their general affective well-being. Moreover, momentary positive affective states were significantly higher after PA sessions, especially after fitness sessions. For both activities, momentary negative affect significantly decreased after PA sessions, with a larger reduction in the levels of high-activated unpleasant affect. In the short and middle terms, brief PA programmes seem relevant to enhance employees' affective well-being. PA programme characteristics are finally discussed.
\end{abstract}

Keywords: Psychological well-being; affective states; physical activity; organizational interventions; work 
Physical activity and employees' affective well-being

\section{Introduction}

According to a recent French National Research and Safety Institute (2016) survey of 28,000 workers from all occupational categories, only $46 \%$ feel fulfilled, $25 \%$ regularly hide their emotions at work and pretend to be in a good mood, 50\% report interacting with individuals in distress or needing to be calmed down, and $21 \%$ to $44 \%$ report tensed working situations (Beque, Kingsada, \& Mauroux, 2019; Coutrot, 2018). Such findings highlight the challenges for working people to maintain a good mental health, defined as "a state of wellbeing in which the individual realises his or her own abilities, can cope with the normal stresses of life, can work productively and fruitfully, and is able to make a contribution to his or her community" (World Health Organization, 2004).

Well-being is a multi-faceted construct referring to optimal psychological functioning and experience (Ryan \& Deci, 2001). This concept has been extensively studied across hedonic and eudaimonic perspectives. In the hedonic approach that focusses on happiness (Diener, 1984; Kahneman et al., 1999), subjective well-being encompasses affective (i.e., momentary positive and negative affective states that occur at different frequencies and intensities through time) and cognitive (i.e., life or life-specific domains satisfaction) components. Regarding the eudaimonic approach of well-being (Ryan \& Deci, 2001) also known as psychological well-being, well-being refers to self-fulfillment and relates on one's resources and strengths, as well as on life meaning, authenticity, and purposefulness (Waterman et al., 2010). Even if affects are not at the core of eudaimonia, both concepts appear correlated (Di Fabio \& Palazzeschi, 2015; Huta \& Ryan, 2010).

Affective states have been defined as states that are accessible to the consciousness (such as feelings) varying over time along two fundamental dimensions: Valence (i.e., positive-negative) and arousal (i.e., high-low activation; Yik, Russell, \& Steiger, 2011). Hence, they may be characterised in terms of high-activated positive affect (HAPA; e.g., excited), low-activated positive affect (LAPA; e.g., peaceful), low-activated negative affect 
(LAUA; e.g., bored), and high-activated negative affect (HAUA; e.g., anxious). A positive ratio of positive to negative affect, is positively associated with mental health status (Diehl, Hay, \& Berg. 2011). According to the broaden-and-build theory (Fredrickson, 2001), individuals who experience frequent positive emotions broaden their thought-action repertoire that enable them to build psychological resources. Affect and affect regulation strategies reciprocally influence one another in such a way that positive affect enhances the use of adaptive affective regulation strategies (e.g., problem-focused coping or cognitive reappraisal) which in turn rise positive affect (Garland, Gaylord, \& Fredrickson, 2011; Pavani, Le Vigouroux, Kop, Congard, \& Dauvier, 2015). In the context of work, positive affect is positively related to job satisfaction, job performance (Lyubomirsky, King, \& Diener, 2005; Wright \& Cropanzano, 2000), and work engagement (Gillet, Becker, Lafrenière, Huart, \& Fouquereau, 2017). In addition, a positive ratio of positive to negative affect is negatively related to cynicism and turnover (Ha, 2018). Although positive affect enables to buffer the effects from a large range of life's stressors (including work stressors) at a daily level (Leger, Charles, \& Almeida, 2020), higher levels of negative affect and lower levels of positive affect are reported when experiencing higher levels of perceived stress (Wang, Schneider, Schwartz, \& Stone, 2020). In the workplace, negative affect is positively related to productivity loss and emotional exhaustion (Ferreira, da Costa Ferreira, Cooper, \& Oliveira, 2019), and negatively linked to job satisfaction (Kafetsios \& Zampetakis, 2008). As such, considering positive and negative affect is of main importance for employees' flourishing as well as for managers.

Physical activity (PA) appears useful to enhance employees' general affective wellbeing as well as momentary affective states. On the one hand, longitudinal studies investigating the effect of PA practice on employees' mental health mainly focused on workrelated indicators such as levels of burnout, stress, anxiety, and depression (Jonsdottir et al., 
Physical activity and employees' affective well-being

2010; Lindwall, Gerber, Jonsdottir, Börjesson, \& Ahlborg, 2014; de Vries et al., 2016) and did not assess affective well-being as a main outcome. Across one to four years, results from these studies report that workers being lightly to vigorously active or increasing their PA level across time are less likely than sedentary workers to report symptoms of stress, depression, and burnout, suggesting a better affective well-being. Only a few interventional studies have investigating the benefits of PA practice on employees' affective well-being as the main outcome in the workplace. Specifically, Hartfiel, Havenhand, Khalsa, Clarke, and Krayer (2011) found that after a six-week yoga programme, employees in the intervention group felt significantly more serene, less sad, and less hostile than those in the control group. In the same lines, Bretland and Thorsteinsson (2015) showed significant improvement in positive and negative affective states and emotional exhaustion after four weeks of PA practice.

On the other hand, results of daily studies reveal that PA's acute effect efficiently enhances employees' momentary positive affective states starting immediately after the practice and continuing for the next few hours (Feuerhahn, Sonnentag, \& Woll, 2014; Ménard, Foucreault, Stevens, Trépanier, \& Flaxman, 2017). Findings about PA’s effect on momentary negative affective states are mixed, with some studies showing a decrease in negative affect following PA (Ménard et al., 2017; Puterman, Weiss, Beauchamp, Mogle, \& Almeida, 2017) while others do not (Feuerhahn et al., 2014; Liao, Shonkoff, \& Dunton, 2015). In the workplace, only a few interventional studies investigated the benefits of PA practice on employees' affective states. Specifically, Thøgersen-Ntoumani et al. (2015) showed that employees being involved in a 10-week walking programme experienced higher levels of enthusiasm and relaxation at work on afternoons when they carried out PA during their lunch breaks compared with days when they did not. Interestingly, de Vries et al. (2020) showed that pleasure experienced during PA sessions moderates the trajectories of health and 
Physical activity and employees' affective well-being

positive affect among employees involved in a six-week running programme. Finally, a recent narrative review emphasizes a positive relationship between acute affective responses to PA and the maintenance of active behaviours (Steven et al., 2020). Precisely, positive affective experiences during PA sessions increase subsequent PA behaviours among low active adults while negative affective experiences would decrease them. Even less is known about a larger influence of affective responses during versus after PA sessions, such effects seem in line with a perspective of hedonic motivations towards PA, in such a way that more positive (or less negative) affective responses during PA is associated with a greater likelihood of future PA behaviour (Steven et al., 2020).

Although relevant, these studies present shortcomings that need to be overcome. First, although general affective well-being assessments and momentary affective states are complementary to examine the process through which the acute effects of PA sessions might account for the broader effects of a whole intervention (Russell \& Daniel, 2018; de Vries et al., 2020), this consideration has received little interest. To date, no previous study has examined the benefits of a PA programme on employees' general affective well-being as well as on their momentary affective states. Regarding employees' mental health, Gerber et al. (2013) examined both timeframes in a pilot study investigating the effect of a twelve-week PA programme including two to three PA sessions per week on employees with high levels of burnout. Nevertheless, the beneficial acute effects of PA practice on momentary affective states momentary affective states were only assessed from before to after two PA sessions and for a half of participants. Considering the upward spiral of positive affective states on well-being (Fredrickson \& Joiner, 2018) as well as the acute effect of PA sessions on affective states, both timeframes need to be further examined.

Furthermore, the duration of PA programmes can also be risen. When wishing to improve employees' affective well-being, intervention studies range from 4 to 12 weeks and 
Physical activity and employees' affective well-being

from 4 to 18 weeks for other work-related variables such as burnout (see Naczenski et al., 2017 for a review). To the best of our knowledge, only one intervention study relied on a four-week PA programme (Bretland \& Thorsteinsson, 2015). Yet, short non-sporting interventions designed to improve affective well-being in employees such as positive psychology interventions (Rahm, Heise, \& Schuldt, 2017), well-being games (Keeman, Näswall, Malinen, \& Kuntz, 2017) or mindfulness (Pavani et al., 2020), show significant benefits from four weeks of practice. Considering the advantages employees and managers can reap from developing short efficient interventions, the promising results from Bretland and Thorsteinsson (2015) need to be replicated and extended with larger sample sizes.

Finally, results from these studies do not indicate if a particular type of PA or intensity of practice should be prioritised to optimize the benefits of PA sessions on subsequent momentary affective states. Previous studies found that light intensity PA such as walk and yoga (Hartfiel et al., 2012; Thøgersen-Ntoumani et al. 2015) as well as moderateto-vigorous PA (e.g., moderate intensity continuous training and high intensity interval) increase HAPA and LAPA post-PA sessions (Oliveira, Santos, Kilpatrick, Pires, \& Deslandes, 2018; Oliveira, Slama, Deslandes, Furtado, \& Santos, 2013). Moreover, the level of effort weekly experienced during PA sessions did not significantly contribute to positive affect nor health-related trajectories among employees involved in a six-week running programme (de Vries et al. 2020). Nevertheless, moderate-to-vigorous PA intensity may have the disadvantage of generating negative affect due to demanding physiological adaptations (Oliveira et al., 2013; Oliveira et al., 2018). Since light to vigorous PA intensities are positively linked with subsequent positive affective states and because variety during PA sessions is associated with greater enjoyment (Juvancic-Heltzel, Glickman, \& Barkley, 2013; Sylvester et al., 2014), varying the nature and intensities between PA sessions appear worthy to investigate when designing a short PA programme. 
Physical activity and employees' affective well-being

Therefore, the present study main aim was to examine the effects of a four-week PA programme on employees' general affective well-being as well as on momentary affective states. We hypothesized that employees who were involved in a four-week PA programme would see significant increases in their general affective well-being during a one-month period in comparison to a comparison group. We also assumed that both PAs would significantly increase momentary positive affective states (HAPA and LAPA) and decrease momentary negative affective states (HAUA and LAUA).

The second purpose was to investigate the acute effects of two different PAs with varied intensities of practice (i.e., fitness activities with a moderate-to-vigorous practice intensity, and stretching activities with a light practice intensity) on momentary affective states. However, we have left as an open research question whether these effects may vary as a function of the type of practice (fitness or stretching).

\section{Materials and Methods}

\subsection{Participants and procedures}

An a-priori test calculation for F tests (i.e., ANOVA: Repeated measures, withinbetween interaction) was run with GPower@. The test calculation was run for a small effect size of .17 (on the basis of previous study working in the benefits of PA on emotional exhaustion; de Vries et al., 2017), a threshold of significance at .05 , a power of .90 , two groups, two measurements, and a correlation of .50 across repeated measures, indicated a requested total sample size of 88 . A rate of potential dropout was estimated at $20 \%$ per group. Therefore, a minimum of 53 participants had to be recruited per group. The study participants were 136 employees from five French service companies. The participants were divided into two groups: An experimental group composed of 79 tertiary employees (65 women and 14 men; $M_{\text {age }}=40.10$ years old, $\left.S D=9.70\right)$ and a comparison group composed of 57 tertiary employees (36 women and 21 men; $M_{\text {age }}=37.72$ years old, $S D=14.23$ ). The study design 
was quasi-experimental since we could not reach for random assignments. Even experimental design would have been preferable, we faced time constraints due to four-week collaborations with our partner companies. In such a way, no waiting list of participants could have been provided. Therefore, employees interested in practicing PA two times a week during four weeks were included in the experimental group whereas employees interested in participating to the study without practicing PA were included in the comparison group.

All participants completed a four-week research programme that included the completion of online measures of general affective well-being one month apart. Participants from the experimental group had to reply to the general affective well-being measures within the respective week before and after the week of the PA programme to avoid overlapping between contextual and situational measures.

Over four weeks, the experimental group participated in a PA programme that included two PA sessions per week supervised by two sports educators. Participants in the comparison group did not modify their regular work schedules. PA sessions were programmed during the lunch break or at the end of the workday, depending on the companies' ability to accommodate the sessions. Each week, the PA programme included two 45-minute sessions that differed in terms of nature and intensity (i.e., moderate to vigorous intensity for the fitness activities and light intensity for the stretching activities). The fitness practice included various exercises such as muscular reinforcement exercises and circuit-training as well as collaborative and entertaining challenges. Meanwhile, the stretching practice was comprised of soft and relaxing exercises that stretched the muscles.

Participants' momentary affective states were assessed with visual analogue scales before and after each session. PA educators made sure all items were filled in each time. Prior to engaging in the PA programme, participants from the experimental group were informed about any health risks that might be associated with PA. 
In order to be eligible for inclusion in the study, participants had to work at least 16 hours per week, and could not be involved in elite competitive sports activities nor having any known health issues such as chronic diseases or traumatic injuries. Finally, participants from the experimental group who could not complete a minimum of $75 \%$ of the PA sessions (i.e., six out of eight PA sessions, ensuring two completed sessions per activity type) were excluded from the analyses. This criterion was recently used by Nymberg et al. (2018), who assumed that a higher proportion of attendance enhances the effect of training. Twenty-five participants from the experimental group were excluded from the analyses. Informed consent was obtained from all participants who were included in the study. All procedures performed in the study were in accordance with the 1964 Helsinki Declaration and its amendments.

Companies interested in developing PA sessions within their organization were contacted by to co-investigators of the present study to introduce the research project and the PA programme.

Participants' recruitment was made in different steps. First, a collaboration was made between the two research laboratories and a company specialized in adapted physical activity whose aim is to develop and propose a large array of PA practices (e.g., fitness, stretching, nordic walking) adapted to various public including companies. Companies that accepted to collaborate communicated to their employees about the possibility for them to freely practice muscular reinforcement and stretching one time per week during four weeks as a pilot before considering a longer-term perspective. A letter of information was sent to the employees to introduce the company and the study, but also to answer to any questions about the nature of PA sessions and/or organizational aspects. At this occasion, they were also informed that a scientific project about the potential effects of the PA sessions on their general well-being would be conducted. Employees were informed that their managers did not order the study and that they were free to be part of the comparison or experimental group. Nevertheless, 
Physical activity and employees' affective well-being

employees who only wished to discover the practice via non-regular PA sessions were allowed to do it but were not included in the study. Employees were not pushed to participate neither by their director nor by the PA educators. There was no conflict of interest between our research laboratories and the different companies. Finally, and at managers and employees' requests, results from the experiment were communicated during meetings.

\subsection{Measures}

General affective well-being: The French version of the Scale of Positive and Negative Experience (SPANE; Martin-Krumm et al., 2017) was used to assess the frequency of participants' positive and negative affective feelings at the beginning and end of the programme. Participants were asked to indicate the extent to which they experienced 12 different general (i.e., pleasant, unpleasant, positive, negative) and specific (e.g., happy, joyful, contented, angry, sad, afraid) feelings during the last four weeks on a five-point unipolar Likert-scale ranging from 1 (very rarely or never) to 5 (very often or always). The SPANE allowed us to compute a score ranging from 6 to 30, representing positive and negative general affective well-being. The scale also facilitated the calculation of an affect balance score ranging from -24 to 24 by subtracting positive scale scores from negative scale scores. Cronbach's alphas were adequate for each affective scale at each assessment time (T1: Before the four-week programme and T2: Four weeks later): $\alpha_{\text {positive feelings_T1 }}=.87$, $\alpha_{\text {positive feelings_T2 }}=.88, \alpha_{\text {negative feelings_T1 }}=.84$, and $\alpha_{\text {negative feelings_T2 }}=.88$.

Momentary affective states: Before and after each PA session, participants in the experimental group were asked to indicate how they felt at the moment on visual analogue scales ranging from 0 (not at all) to 10 (extremely). Four affective states, equally distributed in terms of valence and arousal (i.e., joyful, satisfied, angry, and sad) were selected. These four items were respectively considered as HAPA, LAPA, HAUA, and LAUA. Such choices 
Physical activity and employees' affective well-being

relied on the basis of the Job-Related Affective Well-Being Scale (Van Katwick et al., 2000) that assesses affective states commonly experienced at work by employees.

\subsection{Data analyses}

Data were analysed using Jamovi ${ }^{\circledR}$ software and the General Analyses for Linear Models in Jamovi (GAMLj) module. Regarding general affective well-being analyses, normality tests (i.e., skewness coefficients and analyses of the residuals) revealed that only the affect balance score followed a normal distribution at T1 and T2. Therefore, a linear mixed-model analyses of variance (LMM) was chosen for the study of the affect balance score, while generalized linear mixed model analyses of variance (GLMM) were used for the respective studies of the positive and negative scales. For the positive and negative scales, analyses were performed with Gamma and Inverse Gaussian distributions that both constitute relevant functions to manage unimodal skewed distributions with continuous responses (Lo \& Andrews, 2015). The use of the Gamma function was finally selected on the basis of the AIC criterion (Lo \& Andrews, 2015). Each of the three models had two factors: Time (T1 and T2) and group (experimental and comparison groups), and random intercepts on the cluster variable (subjects). LMM and GLMM were selected since they take the hierarchical structure of the data into account (Noël, 2015). For LMM, pseudo- $R^{2}$ can be interpreted with the use of R-squared marginal corresponding to the variance explained by the fixed effects over the total expected variance of the dependent variable and the R-squared conditional corresponding to the variance explained by the fixed and the random effects together over the total (expected) variance of the dependent variable (Johnson, 2014). For GLMM, effect size can be reported with the odd ratio $(\exp (B))$ of the effects, that enables to interpret the effects in terms of rate of change in the dependent variable as the independent variable increases. General affective well-being analyses were conducted according to intention-to-treat (i.e., referring to the effect of treatment « as assigned » regardless of dropout or missing values) 
and per-protocol (i.e., referring to the effect of treatment « as received », in our case excluding participants who had less than $75 \%$ adherence rate to the PA programme) principles.

A second set of analyses was performed on the data from participants in the experimental group that reached the compliance rate to assess PA sessions' situational effects on momentary affective states. Normality analyses revealed that all the within-subjects conditions for momentary positive affective states followed a normal distribution but that momentary negative affective states' distributions were skewed for almost all conditions (i.e., skewness coefficients being all above .50). In order to satisfy repeated-measures analyses of variance (ANOVA) normality assumptions, a square root transformation was applied to each within-subjects condition. Repeated-measures ANOVA were then conducted with two timelevels (before and after the PA sessions) and two PA activities (fitness and stretching) as within-subject factors for momentary positive and negative affective states. To fine-grain the analyses, repeated-measures ANOVA with two arousal levels (high- and low-activated affective states), two time levels (before and after the PA sessions), and two PA activities (fitness and stretching) as within-subject factors were finally run. In cases of significant effects, Bonferroni post-hoc analyses were used to highlight significant differences across time and groups.

\section{Results}

\subsection{The PA programme's effects on general affective well-being}

Table 1 presents the studied variables' means and standard deviations, while Table 2 presents the fixed effects parameters and random components for each subscale, for perprotocol analyses.

* Insert Table 1 here * 
Physical activity and employees' affective well-being

Per-protocol analyses did not reveal significant main effects of time nor group on the frequency of positive feelings, but revealed a significant time $\times$ group effect $(B=1.49,95 \%$ CI $[0.63,2.35], \exp (b)=4.44)$. According to the odd ratio, the odds for positive feelings to improve across time were four times higher in the experimental group. Post-hoc analyses showed a between-group difference at $\mathrm{T} 1$ with the experimental group having less positive feelings than the comparison group $\left(M_{\mathrm{T} 1_{-} \text {comparison group }}-M_{\mathrm{T} 1_{-} \text {experimental group }}=1.42\right)$. Post-hoc analyses also revealed that the comparison group significantly decreased the frequency of its positive feelings between $\mathrm{T} 1$ and $\mathrm{T} 2\left(M_{\mathrm{T} 2-\mathrm{T} 1}\right.$ comparison group $\left.=-.75\right)$, while the experimental group increased it $\left(M_{\mathrm{T} 2-\mathrm{T} 1 \_ \text {experimental group }}=.74\right)$.

Results also revealed that time exerted a significant effect on the frequency of negative feelings, with a significant decrease in negative feelings across time ( $\mathrm{B}=-1.00,95 \%$ CI $[-1.55,-0.48], \exp (b)=.37)$. The time $\times$ group interaction was significant $(B=-1.62,95 \%$ CI $[-2.64,-0.60], \exp (b)=.20)$, with a significant decrease in negative feelings only for employees in the experimental group $\left(M_{\mathrm{T} 2-\mathrm{T} 1 \_ \text {experimental group }}=-1.81, M_{\mathrm{T} 2-\mathrm{T} 1}\right.$ comparison group $=-$ .19). The main effect of group was not significant. According to the odd ratio, the odds for negative feelings to increase across time were five times lower for participants in the experimental group.

Finally, the results did not reveal significant main effects of time nor group on the affect balance score, but revealed a significant time $\times$ group interaction effect $(B=2.71,95 \%$ CI $[0.81,0.62], R$-marginal $=.02, R$-conditional $=.66)$. Post-hoc analyses showed that only the experimental group significantly increased its ratio of positive to negative feelings one month apart $\left(M_{\mathrm{T} 2-\mathrm{T} 1 \_ \text {experimental group }}=2.24, M_{\mathrm{T} 2-\mathrm{T} 1_{-} \text {comparison group }}=-.48\right)$.

* Insert Table 2 here *

In comparison with per-protocol analyses, intention-to-treat analyses also revealed a significant time $*$ group interaction for positive feelings with a smaller odd ratio $(\mathrm{B}=1.30$, 
Physical activity and employees' affective well-being

95\% CI $[0.51,2.09], \exp (b)=3.68)$; a significant time * group interaction for negative feelings with a larger odd ratio $(B=-2.37,95 \% \mathrm{CI}[-3.48,-1.25], \exp (\mathrm{b})=.09)$; and a significant time $*$ group interaction on the affect balance score with a rather similar effect size $(\mathrm{B}=3.18,95 \% \mathrm{CI}[1.32,5.05], R$-marginal $=.03, R$-conditional $=.61)$.

\subsection{PA sessions' acute effects on momentary affective states}

Table 3 presents the studied variables' means and standard deviations. All effects are detailed in Table 4.

* Insert Table 3 here*

\subsubsection{PA sessions' acute effects on momentary positive affective states}

The results revealed that time $\left[F(1,53)=128.20, p<.001, \eta^{2}{ }_{p}=.71\right]$, type of PA $\left[F(1,53)=19.29, p<.001, \eta^{2}{ }_{p}=.27\right]$, and time $\times$ type of $\mathrm{PA}\left[F(1,53)=20.47, p<.001, \eta^{2}\right.$ $=.28]$ exerted significant effects on momentary positive affect. Interestingly, post-hoc analyses showed that while momentary positive affect was not significantly different before a fitness or a stretching session $\left(M_{\text {positive affect_pre-fitness session }}-M_{\text {positive affect_pre-stretching session }}=.14\right)$, it was significantly different after the PA session, with a larger increase in positive affect after a fitness session than after a stretching session $\left(M_{\text {positive affect_post-fitness session }} M_{\text {positive affect_pre-fitness }}\right.$

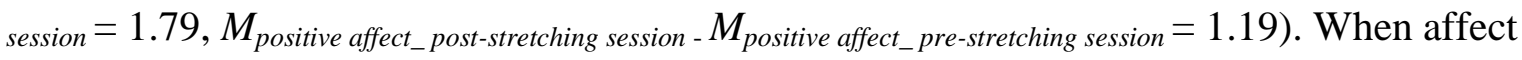
arousal level was factored into the analyses, the results confirmed that time, type of PA, and time $\times$ type of PA (see Table 4 for details) exerted significant effects, but they also showed a significant arousal level $\times$ time interaction $\left[F(1,53)=92.35, p<.001, \eta^{2}{ }_{p}=.64\right]$. Post-hoc analyses revealed that LAPA was significantly higher after the PA sessions ( $M_{\text {LAPA_post-PAsession }}$ - $\left.M_{\text {LAPA_pre-PA session }}=2.77\right)$ while change in HAPA across time was not $\left(M_{\text {HAPA_post-PA session }}\right.$ $\left.M_{\text {HAPA_pre-PA session }}=.21\right)$. The time $\times$ arousal level $\times$ type of PA interaction was not found to be significant.

3.2.2. PA sessions' acute effects on momentary negative affective states 
The results revealed that time $\left[F(1,53)=113.83, p<.001, \eta^{2}{ }_{p}=.68\right]$ exerted a significant effect, with lower negative affect at the end of the PA sessions $\left(M_{\text {sqrt_negative affect_ }}\right.$ post session - $\left.M_{\text {sqrt_negative affect_pre session }}=.41\right)$. Effects related to the type of PA $[F(1,53)=.09, p>$ $.05]$ and time $\times$ type of PA $[F(1,53)=.65, p>.05]$ were beyond the significance level. When affect arousal level was factored into the analyses, the results confirmed that time (see details in Table 4) exerted a significant effect, also revealing significant interaction effects exerted by time $\times$ arousal $\left[F(1,53)=10.93, p<.01, \eta^{2}{ }_{p}=.17\right]$ and arousal level $\times$ type of PA $[F(1$, $\left.53)=6.26, p<.05, \eta^{2}=.10\right]$. The time $\times$ arousal level $\times$ type of PA interaction was not significant. Of particular interest, post-hoc analyses revealed that, while HAUA and LAUA were both significantly different before and after the PA session $\left(M_{\text {square root_HAUA_post-session - }}\right.$

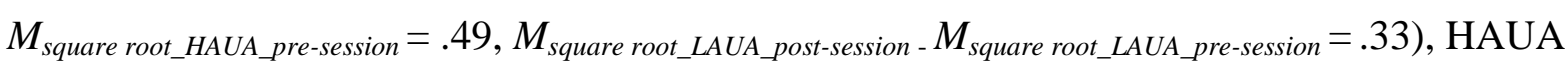
tended to decrease more than LAUA after the PA sessions.

* Insert Table 4 here *

\section{Discussion}

This study's objectives were twofold. Firstly, the study investigated the extent to which a four-week PA programme enhanced employees' general affective well-being within a one-month timeframe. Secondly, the PA sessions' acute effects on momentary positive and negative affect were studied with attention to the time (i.e., pre- versus post-PA practice), the type of PA (i.e., fitness versus stretching), and affective arousal (i.e., high- versus lowactivated).

The first hypothesis found support in the current results, which showed a significant interaction between group and time on positive and negative feelings, highlighting that only employees in the experimental group slightly improved the frequency of its positive feelings and slightly reduced the frequency of its negative feelings after one month. These results reaffirm the effectiveness of PA when interested in enhancing general affective well-being in 
Physical activity and employees' affective well-being

employees (Bretland \& Thorsteinsson, 2015; Hartfiel et al., 2012). They also replicate and extend other studies' results, emphasizing that short interventions, such as PA programmes (Bretland \& Thorsteinsson, 2015) or positive psychological interventions (Rahm et al., 2017) have significant effects on employees' general affective well-being from one month. As such, even brief PA programmes may constitute an interesting way to counterbalance the negative effects of work-related emotional demands by reducing job-related strain and increasing employees' personal affective resources. Avoiding quasi-experimental design whenever possible, future studies should manipulate PA sessions' frequency and duration over a prescribed number of weeks in order to optimize short interventions. Indeed, participants from the experimental group (whether included in per-protocol or in intention-to-treat analyses) improved significantly their general well-being across one month. Such result indicates a reliable effect of the intervention but also suggests that less compliant participants might have also benefitted from the intervention. In the light of de Vries et al.'s (2020) results highlighting that PA exposure modulates the effect of a PA intervention in positive affect over time, it appears worthy to further investigate the minimal threshold for notifying a significant association between PA practice and changes in general affective well-being. Follow-ups at one to three months would also be useful to investigate the maintenance of such beneficial effects in a longer-term perspective.

It was also hypothesized that PA sessions would significantly increase momentary positive affective states (HAPA and LAPA) and decrease momentary negative affective states (HAUA and LAUA). As expected, analyses confirmed that PA significantly increased HAPA and LAPA and also decreased HAUA and LAUA in such a way that participants from the experimental group felt significantly more joyful and satisfied after a PA session but also less angry and sad. These results are in line with previous studies investigating PA sessions' acute effects on momentary affective states (Feuerhahn et al., 2014; Liao et al., 2015; Ménard 
Physical activity and employees' affective well-being

et al., 2017). Regarding negative affect, our results contrast with Feuerhahn et al. (2014), showing that even when momentary HAUA and LAUA are low before a PA session, they significantly decrease after PA sessions. Interestingly, LAPA had a larger increase than HAPA from before to after the PA sessions, while HAUA decreased significantly more than LAUA after the PA session.

According to the close links between momentary and subsequent affective states (Pavani et al., 2015), these repeated affective post-PA session improvements might partially explain why the experimental group significantly increased the frequency of positive feelings and reduced the frequency of negative one month apart. In addition, while post-PA practice affective improvements might have had interesting professional outcomes for employees from the experimental group, these benefits were probably also extended to their organization. Indeed, results from a multi-study (Warr, Bindl, Parker, \& Inceoglu, 2014) have shown that HAPA is positively linked with proactivity, extra-role contribution, strategic scanning, problem prevention, and proficiency, while LAPA is associated with proficient performance. These multi-study's results also positively linked LAUA with effort and social withdrawals. Finally, it can be argued that the PA sessions were appropriately designed and led.

The significant interaction between time (i.e., pre- versus post-session) and the type of PA practice revealed that fitness sessions generated a larger increase in positive affective states than relaxation sessions. Fitness sessions' specific effects seem to align with studies that have claimed that practice intensity (i.e., light versus moderate-to-vigorous) matters when considering work-related well-being indicators (Gerber et al., 2013, 2017; Jonsdottir et al., 2010). Regarding psychological adaptations, such effects could be explained through the necessity to focus one's attention on internal information during strenuous PA practice, thus 
Physical activity and employees' affective well-being

limiting the possibility of entertaining negative work-related thoughts (Feuerhahn et al., 2014) nor rumination (van Hooff, Benthem de Grave, \& Geurts, 2019).

Since, the present study did not objectively control practice intensity, the use of objective monitors, such as heart rate monitors, to control for practice intensity would have been necessary to support this hypothesis. The entertaining content of some fitness exercises (i.e., playfully performed between-group challenges) might also explain why fitness sessions generated more joy and satisfaction than stretching sessions. Indeed, fun experiences are positively related to HAPA and LAPA (Reis, O'Keefe, \& Lane, 2017). Therefore, not only practice intensity but also exercise content might contribute to positive momentary affective states. Future studies should test this hypothesis by manipulating PA session content. Overall, even though both fitness and stretching sessions significantly improved positive momentary affective states, one PA practice's greater effect constitutes an interesting phenomenon that future studies should investigate. Indeed, when viewed as a daily or monthly choice, selecting one PA practice from a large range of possibilities could have specific implications on both employees' affective well-being and organizational needs and goals.

Beyond these results, this study has general limitations.

Firstly, PA sessions were scheduled either at midday or at the end of the workday, according to companies' organizational and technical characteristics (e.g., the duration of lunch breaks, the presence or absence of a worksite shower). Therefore, even though PA sessions were proposed in the workplace setting and were restricted to employees, it cannot be strictly said that PA sessions occurred in the context of work. These elements highlight the need for a better control when scheduling PA sessions in the workplace.

Second, the lack of measures about the different mechanisms that could explain the process through which employees from the experimental group improved their general and momentary affective well-being appears as a limitation. As a process, recovery experiences 
Physical activity and employees' affective well-being

that enable the psychological and physiological systems activated due to job demands or other stressors to return to their pre-demand levels (Sonnentag \& Geurts, 2009) via psychological detachment from work, relaxation, mastery, and control, should have been assessed. Indeed, psychological detachment have been found to partially mediate the relationship between PA practiced after work and positive affect in the following evening (Feuerhahn et al., 2014), and to be improved during high-intensity PA practice (van Hooff et al., 2019). Positive physical self-perceptions including the feeling of competence are also recognized as a partial mediator of the relationship between PA practiced after work and positive affect in the following evening (Feuerhahn et al., 2014). Even if a short PA programme of the two different practices might appear rather short to develop new skills in one month, experiencing challenging situations leading to different forms of achievement (e.g., number of repetition done), might have partially contributed to the enhancement of positive affect. The sense of belonging which also constitutes a mediator of the relationship between PA practiced after work and positive affect in the evening (Feueurhan et al., 2014) might have been investigated since the quantity and quality of social interactions (Sun, Harris, \& Vazire, 2019) are positively linked with affective well-being. Third, only four momentary affective states were selected on the basis of reliable tools and theoretical models (Van Katwick et al., 2000; Warr et al., 2014; Yik et al., 2011). Nevertheless, due to the respective five degrees of negative and positive affect according to their arousal level, further research is encouraged to examine in more details the relationship between physical activity and momentary affective states.

Moreover, individuals' affective states and well-being are influenced by several workrelated factors, such as workload and organizational constraints (Van Katwyk, Fox, Spector, \& Kelloway, 2000). Although they were not of primary interest, controlling for their respective contributions could have helped clarify the PA programme's effects on affective 
Physical activity and employees' affective well-being

well-being. Finally, the use of a quasi-experimental design does not allow concluding to a causal relationship between the completion of the PA programme and changes in affective well-being across one month. As recently pointed out, the difficulty in randomly allocating participants in organizational settings is a limitation that should be overcome (Ginoux, Isoard-Gautheur, \& Sarrazin, 2019).

\section{Conclusion}

This study is one of the first to examine the contextual and situational effects of a short PA programme on employees' general and acute affective well-being. The results highlight significant improvements in general affective well-being for employees involved in the PA programme. Moreover, PA sessions increased positive affective states and decreased negative affective states. Of particular interest, 1) fitness sessions had a greater effect than stretching sessions on momentary positive affective states, and 2) LAPA was significantly higher than HAPA after PA sessions. Further studies are needed to assess the potential effect of the nature of PA (e.g., individual vs. collective, indoor vs. outdoor practices) as well as the process (e.g., psychological detachment, dose-response) through which general well-being and momentary affective states fluctuate following PA practice. 
Physical activity and employees' affective well-being

\section{References}

Beque, M., Kingsada, A., Mauroux, A. (2019). Conflits de valeurs et exigences émotionnelles (Dares Report Number 28). https://dares.travail-emploi.gouv.fr/dares-etudes-etstatistiques/etudes-et-syntheses/synthese-stat-synthese-eval/article/conflits-de-valeurset-exigences-emotionnelles

Bernard, P., Doré, I., Romain, A. J., Hains-Monfette, G., Kingsbury, C., \& Sabiston, C. (2018). Dose response association of objective physical activity with mental health in a representative national sample of adults: A cross-sectional study. Plos One, 13(10), e0204682. https://doi.org/10.1371/journal.pone.0204682

Bretland, R. J., \& Thorsteinsson, E. B. (2015). Reducing workplace burnout: The relative benefits of cardiovascular and resistance exercise. PeerJ, 3, e891. https://doi.org/10.7717/peerj.891

Coutrot, T. (2018). Travail et bien-être psychologique (Dares Report Number 217). https://dares.travail-emploi.gouv.fr/IMG/pdf/travail_et_bien-etre_tc_vd_2.pdf

Demerouti, E., Bakker, A. B., Nachreiner, F., \& Schaufeli, W. B. (2001). The job demandsresources model of burnout. Journal of Applied Psychology, 86(3), 499-512. https://doi.org/10.1037/0021-9010.86.3.499

Diehl, M., Hay, E. L., \& Berg, K. M. (2011). The ratio between positive and negative affect and flourishing mental health across adulthood. Aging \& Mental Health, 15(7), 882893. https://doi.org/10.1080/13607863.2011.569488

Diener E. (1984). Subjective well-being. Psychological Bulletin, 95, 542-575.

Diener, E., Wirtz, D., Tov, W., Kim-Prieto, C., Choi. D., Oishi, S., \& Biswas-Diener, R. (2010). New measures of well-being: Flourishing and positive and negative feelings. Social Indicators Research, 97, 143-156. https://doi.org/10.1007/s11205-009-9493-y 
Physical activity and employees' affective well-being

Di Fabio, A., \& Palazzeschi, L. (2015). Hedonic and eudaimonic well-being: The role of resilience beyond fluid intelligence and personality traits. Frontiers in Psychology, 6, 1367.

Ferreira, A. I., da Costa Ferreira, P., Cooper, C. L., \& Oliveira, D. (2019). How daily negative affect and emotional exhaustion correlates with work engagement and presenteeism-constrained productivity. International Journal of Stress Management, 26(3), 261-271. https://doi.org/10.1037/str0000114

Feuerhahn, N., Sonnentag, S., \& Woll, A. (2014). Exercise after work, psychological mediators, and affect: A day-level study. European Journal of Work and Organizational Psychology, 23(1), 62-79. https://doi.org/10.1080/1359432X.2012.709965

Fredrickson, B. L. (2001). The role of positive emotions in positive psychology. American Psychologist, 56(3), 218-226. https://doi.org/10.1037//0003-066X.56.3.218

Fredrickson, B. L., \& Joiner, T. (2018). Reflections on Positive Emotions and Upward Spirals. Perspectives on Psychological Science, 13(2), 194-199. https://doi.org/10.1111/1467-9280.00431

Garland, E. L., Gaylord, S. A., \& Fredrickson, B. L. (2011). Positive reappraisal mediates the stress-reductive effects of mindfulness: An upward spiral process. Mindfulness, 2(1), 59-67. https://doi.org/10.1007/s12671-011-0043-8

Gerber, M., Brand, S., Elliot, C., Holsboer-Trachsler, E., Pühse, U., \& Beck, J. (2013). Aerobic exercise training and burnout: A pilot study with male participants suffering from burnout. BMC Research Notes, 6(1), 78. https://doi: 10.1186/1756-0500-6-78.

Gerber, M., Lindwall, M., Lindegård, A., Börjesson, M., \& Jonsdottir, I. H. (2013). Cardiorespiratory fitness protects against stress-related symptoms of burnout and 
Physical activity and employees' affective well-being

depression. Patient Education and Counseling, 93(1), 146-152.

https://doi.org/10.1016/j.pec.2013.03.021

Gerber M, Ludyga S, Mücke M, Colledge F, Brand S, \& Pühse, U. (2017). Low vigorous physical activity is associated with increased adrenocortical reactivity to psychosocial stress in students with high stress perceptions. Psychoneuroendocrinology, 80, 104-113. https://doi:10.1016/j.psyneuen.2017.03.004

Gillet, N., Becker, C., Lafrenière, M.-A., Huart, I., \& Fouquereau, E. (2017). Organizational support, job resources, soldiers' motivational profiles, work engagement, and affect. Military Psychology, 29(5), 418-433. https://doi.org/10.1037/mil0000179

Ginoux, C., Isoard-Gautheur, S., \& Sarrazin, P. (2019). "Workplace Physical Activity Program" (WOPAP) study protocol: a four-arm randomized controlled trial on preventing burnout and promoting vigor. BMC Public Health, 19(1), 289. https://doi.org/10.1186/s12889-019-6598-3

Ha, S. (2018). Surface acting and job-related affective wellbeing: Preventing resource loss spiral and resource loss cycle for sustainable workplaces. Sustainability, 10(4), 1099. https://doi.org/10.3390/su10041099

Hartfiel, N., Havenhand, J., Khalsa, S. B., Clarke, G., \& Krayer, A. (2011). The effectiveness of yoga for the improvement of well-being and resilience to stress in the workplace. Scandinavian Journal of Work, Environment \& Health, 37(1), 70-76. https://doi.org/10.5271/sjweh.2916

van Hooff, M. L. M., Benthem de Grave, R. M., \& Geurts, S. A. E. (2019). No pain, no gain? Recovery and strenuousness of physical activity. Journal of Occupational Health Psychology, 24(5), 499-511. https://doi.org/10.1037/ocp0000141 
Physical activity and employees' affective well-being

Huta, V., \& Ryan, R. M. (2010). Pursuing pleasure or virtue: The differential and overlapping well-being benefits of hedonic and eudaimonic motives. Journal of Happiness Studies, 11(6), 735-762. https://doi.org/10.1007/s10902-009-9171-4

Johnson, P. C.D. (2014). Extension of Nakagawa \& Schielzeth's $\mathrm{R}_{\text {GLMM }}^{2}$ to random slopes models. Methods in Ecology and Evolution, 5, 944-946. https://doi.org/10.1111/2041210X.12225

Jonsdottir, I. H., Rödjer, L., Hadzibajramovic, E., Börjesson, M., \& Ahlborg, G. (2010). A prospective study of leisure-time physical activity and mental health in Swedish health care workers and social insurance officers. Preventive Medicine, 51(5), 373-377. https://doi.org/10.1016/j.ypmed.2010.07.019

Juvancic-Heltzel, J. A., Glickman, E. L., \& Barkley, J. E. (2013). The effect of variety on physical activity: a cross-sectional study. Journal of Strength and Conditioning Research, 27(1), 244-251. https://doi.org/10.1519/JSC.0b013e3182518010

Kafetsios, K., \& Zampetakis, L. A. (2008). Emotional intelligence and job satisfaction: Testing the mediatory role of positive and negative affect at work. Personality and Individual Differences, 44(3), 712-722. https://doi.org/10.1016/j.paid.2007.10.004

Kahneman, D. (1999). Objective happiness. In D. Kahneman, E. Diener, \& N. Schwartz (Eds.), Well-being: The foundations of hedonic psychology (pp. 3-25). New York: Russell Sage.

Keeman, A., Näswall, K., Malinen, S., \& Kuntz, J. (2017). Employee wellbeing: Evaluating a wellbeing intervention in two settings. Frontiers in Psychology, 8, 505. https://doi.org/10.3389/fpsyg.2017.00505

Leger, K.A., Charles, S.T. \& Almeida, D.M. (2020). Positive emotions experienced on days of stress are associated with less same-day and next-day negative emotion. Affective Science, 1, 20-27. https://doi.org/10.1007/s42761-019-00001-w 
Physical activity and employees' affective well-being

Liao, Y., Shonkoff, E. T., \& Dunton, G. F. (2015). The acute relationships between affect, physical feeling states, and physical activity in daily life: A review of current evidence. Frontiers in Psychology, 6. https://doi.org/10.3389/fpsyg.2015.01975

Lindwall, M., Gerber, M., Jonsdottir, I. H., Börjesson, M., \& Ahlborg, G. (2014). The relationships of change in physical activity with change in depression, anxiety, and burnout: A longitudinal study of swedish healthcare workers. Health Psychology, 33(11), 1309-1318. https://doi.org/10.1037/a0034402

Lo, S., \& Andrews, S. (2015). To transform or not to transform: using generalized linear mixed models to analyse reaction time data. Frontiers in Psychology, 6, 1171.

https://doi: 10.3389/fpsyg.2015.01171

Lyubomirsky, S., King, L., \& Diener, E. (2005). The benefits of frequent positive affect: Does happiness lead to success? Psychological Bulletin, 131(6), 803-855. https://doi.org/10.1037/0033-2909.131.6.803

McAuley, E., \& Courneya, K. S. (1994). The subjective exercise experiences scale (SEES): development and preliminary evaluation. Journal of Sport and Exercise Psychology $16,163-177$.

Maddux, R. E., Daukantaité, D., \& Tellhed, U. (2018). The effects of yoga on stress and psychological health among employees: An 8- and 16-week intervention study. Anxiety, Stress, and Coping, 31(2), 121-134. https://doi.org/10.1080/10615806.2017.1405261

Martin-Krumm, C., Fenouillet, F., Csillik, A., Kern, L., Besancon, M., Heutte, J., Paquet, Y., Delas, Y., Trousselard, M., Lecorre, B., \& Diener, E. (2017). Changes in emotions from childhood to young adulthood. Child Indicator Research, 11, 541-561. https://doi.org/10.1007/s12187-016-9440-9 
Physical activity and employees' affective well-being

Ménard, J., \& Foucreault, A., Stevens, C., Trépanier, S.-G., \& Flaxman, P., (2016). Daily fluctuations in office-based workers' leisure activities and Well-Being. International Journal of Psychological Studies, 9(1), 47. https://doi.org/10.5539/ijps.v9n1p47

Naczenski, L. M., de Vries, J. D., van Hooff, M. L. M., \& Kompier, M. A. J. (2017). Systematic review of the association between physical activity and burnout. Journal of Occupational Health, 59(6), 477-494. https://doi.org/10.1539/joh.17-0050-RA

Noël, Y. (2015). Modèles linéaires généralisés à effets fixes et aléatoires de la variabilité inter et intra-individuelle. In J. Juhel \& Rouxel G. (Eds), Différences et variabilités en psychologie (pp. 237-264). Rennes: Presses Universitaires de Rennes.

Nymberg, P., Ekvall Hansson, E., Stenman, E., Calling, S., Sundquist, K., Sundquist, J., \& Zöller, B. (2018). Pilot study on increased adherence to physical activity on prescription (PAP) through mindfulness: Study protocol. Trials, 19(1), 563. https://doi.org/10.1186/s13063-018-2932-9

Oliveira, B., Santos, T. M., Kilpatrick, M., Pires, F. O., \& Deslandes, A. C. (2018). Affective and enjoyment responses in high intensity interval training and continuous training: A systematic review and meta-analysis. Plos One, 13(6), e0197124. https://doi.org/10.1371/journal.pone.0197124

Oliveira, B. R., Slama, F. A., Deslandes, A. C., Furtado, E. S., \& Santos, T. M. (2013). Continuous and high-intensity interval training: which promotes higher pleasure? Plos One, 8(11), e79965. https://doi.org/10.1371/journal.pone.0079965

Pavani, J.-B., Le Vigouroux, S., Kop, J.-L., Congard, A., \& Dauvier, B. (2015). Affect and affect regulation strategies reciprocally influence each other in daily life: The case of positive reappraisal, problem-focused coping, appreciation and rumination. Journal of Happiness Studies, 17(5), 2077-2095. https://doi.org/10.1007/s10902-015-9686-9 
Pavani, J. B., Berna, G., Andreotti, E., Guiller, T., Antoine, P., Dauvier, B., \& Congard, A. (2020). Between-individual differences in baseline well-being and emotion regulation strategy use moderate the effect of a self-help cognitive-behavioral intervention for typical adults. Applied Psychology: Health and Well-being, 12(2), 411-431. https://doi.org/10.1111/aphw.12189

Pereira, D., Hächler, P., \& Achim, E. (2017). Recovery experiences during vacation and their association with job stressors and health. Escritos de Psychologia, 10(1), 13-30. https://doi.org/10.5231/psy.writ.2017.1001

Puterman, E., Weiss, J., Beauchamp, M. R., Mogle, J., \& Almeida, D. M. (2017). Physical activity and negative affective reactivity in daily life. Health Psychology, 36(12), 11861194. https://doi.org/10.1037/hea0000532

Rahm, T., Heise, E., \& Schuldt, M. (2017). Measuring the frequency of emotions : Validation of the Scale of Positive And Negative Experience (SPANE) in Germany. Plos One, 12(2), e0171288. https://doi.org/10.1371/journal.pone.0171288

Reis, H. T., O’Keefe, S. D., \& Lane, R. D. (2017). Fun is more fun when others are involved. The Journal of Positive Psychology, 12(6), 547-557. https://doi.org/10.1080/17439760.2016.1221123

Russell, E. \& Daniels, K. (2018). Measuring affective well-being at work using short-form scales: Implications for affective structures and participant instructions. Human Relations, 71(11) 1478-1507. https://doi.org/10.1177/0018726717751034

Ryan, R. M., \& Deci, E. L. (2001). To be happy or to be self-fulfilled: a review of research on hedonic and eudaimonic well-being. Annual Review of Psychology, 52, 141-166. 
Physical activity and employees' affective well-being

Sonnentag, S., Venz, L., \& Casper, A. (2017). Advances in recovery research: What have we learned? What should be done next? Journal of Occupational Health Psychology, 22(3), 365-380. https://doi.org/10.1037/ocp0000079

Stevens, C. J., Baldwin, A. S., Bryan, A. D., Conner, M., Rhodes, R. E., \& Williams, D. M. (2020). Affective determinants of physical activity: A conceptual framework and narrative review. Frontiers in psychology, 11, 568331.

https://doi.org/10.3389/fpsyg.2020.568331

Sun, J., Harris, K., \& Vazire, S. (2020). Is well-being associated with the quantity and quality of social interactions? Journal of Personality and Social Psychology, 119(6), 14781496. https://doi.org/10.1037/pspp0000272

Sylvester, B. D., Standage, M., Dowd, A. J., Martin, L. J., Sweet, S. N., \& Beauchamp, M. R. (2014). Perceived variety, psychological needs satisfaction and exercise-related wellbeing. Psychology \& Health, 29(9), 1044-1061. https://doi.org/10.1080/08870446.2014.907900

Thøgersen-Ntoumani, C., Loughren, E. A., Kinnafick, F.-E., Taylor, I. M., Duda, J. L., \& Fox, K. R. (2015). Changes in work affect in response to lunchtime walking in previously physically inactive employees : A randomized trial. Scandinavian Journal of Medicine \& Science in Sports, 25(6), 778-787. https://doi.org/10.1111/sms.12398

Van Katwyk, P. T., Fox, S., Spector, P. E., \& Kelloway, E. K. (2000). Using the Job-related Affective Well-being Scale (JAWS) to investigate affective responses to work stressors. Journal of Occupational Health Psychology, 5(2), 219-230. https://doi.org/10.1037//1076-8998.5.2.219

de Vries, J. D., Claessens, B. J. C., van Hooff, M. L. M., Geurts, S. A. E., van den Bossche, S. N. J., \& Kompier, M. A. J. (2016). Disentangling longitudinal relations between physical activity, work-related fatigue, and task demands. International Archives of 
Physical activity and employees' affective well-being

Occupational and Environmental Health, 89(1), 89-101.

https://doi.org/10.1007/s00420-015-1054-X

de Vries, J. D., van Hooff, M. L. M., Geurts, S. A. E., \& Kompier, M. A. J. (2020). Process evaluation of the receipt of an exercise intervention for fatigued employees: the role of exposure and exercise experiences. European Journal of Work and Organizational Psychology. https://doi.org/10.1080/1359432X.2020.1829034

de Vries, J. D., van Hooff, M. L. M., Geurts, S. A. E., \& Kompier, M. A. J. (2017). Exercise to reduce work-related fatigue among employees : A randomized controlled trial. Scandinavian Journal of Work, Environment \& Health, 43(4), 337-349. https://doi.org/10.5271/sjweh.3634

Wang, D., Schneider, S., Schwartz, J. E., \& Stone, A. A. (2020). Heightened stress in employed individuals is linked to altered variability and inertia in emotions. Frontiers in Psychology, 11, 1152. https://doi.org/10.3389/fpsyg.2020.01152

Warr, P., Bindl, U. K., Parker, S. K., \& Inceoglu, I. (2014). Four-quadrant investigation of job-related affects and behaviours. European Journal of Work and Organizational Psychology, 23(3), 342-363. https://doi.org/10.1080/1359432X.2012.744449

Waterman, A. S., Schwartz, S. J., Zamboanga, B. L., Ravert, R. D., Williams, M. K., Bede Agocha, V., et al. (2010). The questionnaire for eudaimonic well-being: Psychometric properties, demographic comparisons, and evidence of validity. The Journal of Positive Psychology, 5, 41-61. https://doi: 10.1080/17439760903435208

World Health Organization (2004). Promoting mental health: Concepts, emerging evidence, practice. https://www.who.int/mental_health/evidence/en/promoting_mhh.pdf 
Physical activity and employees' affective well-being

Wright, T. A., \& Cropanzano, R. (2000). Psychological well-being and job satisfaction as predictors of job performance. Journal of Occupational Health Psychology, 5(1), 8494. https://doi.org/10.1037/1076-8998.5.1.84

Yik, M., Russell, J. A., \& Steiger, J. H. (2011). A 12-point circumplex structure of core affect. Emotion, 11(4), 705-731. https://doi.org/10.1037/a0023980 